\title{
Stochastic Stability of Uncertain Hopfield Neural Networks with Discrete and Distributed Delays
}

\author{
Zidong Wang ${ }^{a, b, *}$, Yurong $\operatorname{Liu}^{c}, \operatorname{Karl~Fraser}^{a}$, and Xiaohui Liu ${ }^{a}$
}

\begin{abstract}
This paper is concerned with the global asymptotic stability analysis problem for a class of uncertain stochastic Hopfield neural networks with discrete and distributed time-delays. By utilizing a Lyapunov-Krasovskii functional, using the well-known $S$-procedure and conducting stochastic analysis, we show that the addressed neural networks are robustly, globally, asymptotically stable if a convex optimization problem is feasible. Then, the stability criteria are derived in terms of linear matrix inequalities (LMIs), which can be effectively solved by some standard numerical packages. The main results are also extended to the multiple time-delay case. Two numerical examples are given to demonstrate the usefulness of the proposed global stability condition.
\end{abstract}

\section{Index Terms}

Hopfield neural networks; Uncertain systems; Stochastic systems; Distributed delays; Discrete delays; LyapunovKrasovskii functional; Global asymptotic stability; Linear matrix inequality.

\section{INTRODUCTION}

In the past two decades, since its initiation in [12], the well-known Hopfield neural network has been extensively studied, and successfully applied in many areas such as combinatorial optimization, signal processing and pattern recognition, see e.g. [12], [13], [16], [17], [28]. In particular, the stability problem of Hopfield neural networks has received much research attention since, when applied, the neural network is sometimes assumed to have only one equilibrium that is globally stable. On the other hand, axonal signal transmission delays often occur in various neural networks, and may cause undesirable dynamic network behaviors such as oscillation and instability. Therefore, there has been a growing research interest on the stability analysis problems for delayed neural networks, and a large amount of literature has been available. Sufficient conditions, either delay-dependent or delay-independent, have been proposed to guarantee the asymptotic or exponential stability for neural networks, see [1], [6], [7], [15] for some recent results. It is noticed that, so far, most works on delayed neural networks have dealt with the stability analysis problems for neural networks with discrete time-delays.

Traditionally, discrete time-delays in the models of delayed feedback systems serve as a good approximation in simple circuits having a small number of cells. Nevertheless, a neural network usually has a special nature due to

This work was supported in part by the Engineering and Physical Sciences Research Council (EPSRC) of the U.K. under Grant GR/S27658/01, the Nuffield Foundation of the U.K. under Grant NAL/00630/G, and the Alexander von Humboldt Foundation of Germany.

${ }^{a}$ Department of Information Systems and Computing, Brunel University, Uxbridge, Middlesex, UB8 3PH, United Kingdom.

${ }^{b}$ School of Information Sciences and Technology, Donghua University, Shanghai 200051, China.

${ }^{c}$ Y. Liu is with the Department of Mathematics, Yangzhou University, Yangzhou 225002, P. R. China.

${ }^{*}$ Corresponding author. Address: Department of Information Systems and Computing, Brunel University, Uxbridge, Middlesex, UB8 3PH, United Kingdom. Email: Zidong. Wang@brunel.ac.uk, Fax: ++44/1895 251686. 
the presence of an amount of parallel pathways with a variety of axon sizes and lengths. Such an inherent nature can be suitably modeled by distributed delays [19], [21], because the signal propagation is distributed during a certain time period. For example, in [21], a neural circuit has been designed with distributed delays, which solves a general problem of recognizing patterns in a time-dependent signal. Hence, both discrete and distributed delays should be taken into account when modeling neural networks [20]. Recently, there have been some initial studies on the stability analysis issue for various neural networks with distributed time-delays, see [18], [29], [30]. In [29], criteria ensuring the existence, uniqueness, and global asymptotic stability have been derived for Hopfield neural networks involving distributed delays. It should be mentioned that, most recently, the global asymptotic stability analysis problem has been investigated in [24] for a general class of neural networks with both discrete and distributed time-delays, where a linear matrix inequality (LMI) approach has been developed to establish the sufficient stability conditions.

In recent years, the stability analysis issues for neural networks in the presence of parameter uncertainties and/or stochastic perturbations have stirred some initial research attention. The reason is twofold: 1) the connection weights of the neurons depend on certain resistance and capacitance values that include uncertainties (modeling errors), and 2) in real nervous systems the synaptic transmission is a noisy process brought on by random fluctuations from the release of neurotransmitters, and other probabilistic causes. Therefore, the stability properties have been investigated for delayed neural networks with parameter uncertainties (see e.g. [2], [27]) or external stochastic perturbations (see e.g. [3], [14]). However, to the best of the authors' knowledge, the robust stability analysis problem for stochastic Hopfield neural networks with discrete and distributed delays has not been properly addressed, which still remains important and challenging.

In this paper, we deal with the global robust stability analysis problem for a class of stochastic Hopfield neural networks with discrete and distributed time-delays. By utilizing a Lyapunov-Krasovskii functional and using the wellknown $S$-procedure, we recast the addressed stability analysis problem into a convex optimization problem. Different from the commonly used matrix norm theories (such as the $M$-matrix method), a unified linear matrix inequality (LMI) approach is developed to establish sufficient conditions for the neural networks to be robustly, globally, asymptotically stable. Note that LMIs can be easily solved by using the Matlab LMI toolbox, and no tuning of parameters is required [4]. Two numerical examples are provided to show the usefulness of the proposed global stability condition.

Notations: The notations are quite standard. Throughout this paper, $\mathbb{R}^{n}$ and $\mathbb{R}^{n \times m}$ denote, respectively, the $n$ dimensional Euclidean space and the set of all $n \times m$ real matrices. The superscript " $T$ " denotes matrix transposition and the notation $X \geq Y$ (respectively, $X>Y$ ) where $X$ and $Y$ are symmetric matrices, means that $X-Y$ is positive semidefinite (respectively, positive definite). $I_{n}$ is the $n \times n$ identity matrix. $|\cdot|$ is the Euclidean norm in $\mathbb{R}^{n}$. If $A$ is a matrix, denote by $\|A\|$ its operator norm, i.e., $\|A\|=\sup \{|A x|:|x|=1\}=\sqrt{\lambda_{\max }\left(A^{T} A\right)}$ where $\lambda_{\max }(\cdot)$ (respectively, $\lambda_{\min }(\cdot)$ ) means the largest (respectively, smallest) eigenvalue of $A . l_{2}[0, \infty]$ is the space of square integrable vector. Moreover, let $\left(\Omega, \mathcal{F},\left\{\mathcal{F}_{t}\right\}_{t \geq 0}, P\right)$ be a complete probability space with a filtration $\left\{\mathcal{F}_{t}\right\}_{t \geq 0}$ satisfying the usual conditions (i.e., the filtration contains all $P$-null sets and is right continuous). Denote by $L_{\mathcal{F}_{0}}^{p}\left([-h, 0] ; \mathbb{R}^{n}\right)$ the family of all $\mathcal{F}_{0}$-measurable $C\left([-h, 0] ; \mathbb{R}^{n}\right)$-valued random variables $\xi=\{\xi(\theta):-h \leq \theta \leq 0\}$ such that $\sup _{-h \leq \theta \leq 0} \mathbb{E}|\xi(\theta)|^{p}<\infty$ where $\mathbb{E}\{\cdot\}$ stands for the mathematical expectation operator with respect to the given probability measure $P$. The shorthand $\operatorname{diag}\left\{M_{1}, M_{2}, \cdots, M_{N}\right\}$ denotes a block diagonal matrix with diagonal blocks being the matrices $M_{1}, M_{2}, \cdots, M_{N}$. Sometimes, the arguments of a function or a matrix will be omitted in the analysis when no confusion can arise. 


\section{Problem FORMULATiON}

Recently, Hopfield neural networks with time delays, either discrete or distributed, have been widely investigated, and many stability criteria have been established, see e.g. [2], [6], [7], [18], [24], [29] for some recent results. As in [24], the Hopfield neural network with both discrete and distributed delays can be described by the following model:

$$
\dot{u}(t)=-A u(t)+W_{0} g_{0}(u(t))+W_{1} g_{1}(u(t-h))+W_{2} \int_{t-\tau}^{t} g_{2}(u(s)) d s+V
$$

where $u(t)=\left[u_{1}(t), u_{2}(t), \cdots, u_{n}(t)\right]^{T} \in \mathbb{R}^{n}$ is the state vector associated with the $n$ neurons, the diagonal matrix $A=\operatorname{diag}\left(a_{1}, a_{2}, \cdots, a_{n}\right)$ has positive entries $a_{i}>0$. The matrices $W_{0}=\left(w_{i j}^{0}\right)_{n \times n}, W_{1}=\left(w_{i j}^{1}\right)_{n \times n}$ and $W_{2}=$ $\left(w_{i j}^{2}\right)_{n \times n}$ are, respectively, the connection weight matrix, the discretely delayed connection weight matrix, and the distributively delayed connection weight matrix. $g_{i}(u(t))=\left[g_{i 1}\left(u_{1}\right), g_{i 2}\left(u_{2}\right), \cdots, g_{i n}\left(u_{n}\right)\right]^{T}(i=0,1,2)$ denotes the neuron activation function with $g_{i}(0)=0$, and $V=\left[V_{1}, V_{2}, \cdots, V_{n}\right]^{T}$ is a constant external input vector. The scalar $h>0$, which may be unknown, denotes the discrete time delay, whereas the scalar $\tau>0$ is the known distributed time-delay.

In this paper, we make the following assumption for the neuron activation functions.

Assumption 1: The neuron activation functions in (1), $g_{i}(\cdot)$, satisfy the following Lipschitz condition

$$
\left|g_{i}(x)-g_{i}(y)\right| \leq\left|G_{i}(x-y)\right|, \quad \forall x, y \in \mathbb{R} \quad(i=0,1,2)
$$

where $G_{i} \in \mathbb{R}^{n \times n}$ are known constant matrices.

It is traditionally assumed that the activation functions are continuous, differentiable, monotonically increasing and bounded, such as the functions of sigmoid type. In this paper, these restrictions are removed, and only Lipschitz conditions are imposed in Assumption 1. Note that the type of activation functions in (2) have already been used in numerous papers, see [7] and references therein.

Let $u^{*}$ be the equilibrium point of (1). For the purpose of simplicity, we can shift the intended equilibrium $u^{*}$ to the origin by letting $x=u-u^{*}$, and then the system (1) can be transformed into:

$$
\dot{x}(t)=-A x(t)+W_{0} l_{0}(x(t))+W_{1} l_{1}(x(t-h))+W_{2} \int_{t-\tau}^{t} l_{2}(x(s)) d s,
$$

where $x(t)=\left[x_{1}(t), x_{2}(t), \cdots, x_{n}(t)\right]^{T} \in \mathbb{R}^{n}$ is the state vector of the transformed system. It follows from (2) that the transformed neuron activation functions $l_{i}(x)=g_{i}\left(x+u^{*}\right)-g_{i}\left(u^{*}\right)(i=0,1,2)$ satisfy

$$
\left|l_{i}(x)\right| \leq\left|G_{i} x\right|
$$

where $G_{i} \in \mathbb{R}^{n \times n}(i=0,1,2)$ are specified in (2).

As discussed in Section I, in reality, it is often the case that the connection weights of the neurons include uncertainties, and the neural network is disturbed by environmental noises that affect the stability of the equilibrium. In this paper, as in [2], [3], [14], the Hopfield neural network with parameter uncertainties and stochastic perturbations can be described as follows:

$$
\begin{aligned}
d x(t)= & {\left[-\bar{A} x(t)+\bar{W}_{0} l_{0}(x(t))+\bar{W}_{1} l_{1}(x(t-h))+\bar{W}_{2} \int_{t-\tau}^{t} l_{2}(x(s)) d s\right] d t } \\
& +\sigma(t, x(t), x(t-h)) d w(t)
\end{aligned}
$$


where $w(t)=\left[w_{1}(t), w_{2}(t), \cdots, x_{m}(t)\right]^{T} \in \mathbb{R}^{m}$ is a Brownian motion defined on $\left(\Omega, \mathcal{F},\left\{\mathcal{F}_{t}\right\}_{t \geq 0}, P\right)$, the matrices

$$
\bar{A}=A+\Delta A, \quad \bar{W}_{0}=W_{0}+\Delta W_{0}, \quad \bar{W}_{1}=W_{1}+\Delta W_{1}, \quad \bar{W}_{2}=W_{2}+\Delta W_{2},
$$

and

$$
\Delta A=M_{A} F N_{A}, \quad \Delta W_{i}=M_{i} F N_{i}, \quad(i=0,1,2)
$$

where $\Delta A$ is a diagonal matrx, and $M_{A}, N_{A}, M_{i}, N_{i}(i=0,1,2)$ are known real constant matrices with appropriate dimensions, which characterize how the deterministic uncertain parameter in $F$ enters the nominal matrices $A$ and $W_{i}$ $(i=0,1,2)$. The matrix $F$, which may be time-varying, is unknown and satisfies

$$
F^{T} F \leq I .
$$

Assume that $\sigma: \mathbb{R}^{+} \times \mathbb{R}^{n} \times \mathbb{R}^{n}$ is locally Lipschitz continuous and satisfies the linear growth condition ([5], [11]). Moreover, $\sigma$ satisfies

$$
\operatorname{trace}\left[\sigma^{T}(t, x(t), x(t-h)) \sigma(t, x(t), x(t-h))\right] \leq\left|\Sigma_{1} x(t)\right|^{2}+\left|\Sigma_{2} x(t-h)\right|^{2}
$$

where $\Sigma_{1}$ and $\Sigma_{2}$ are known constant matrices with appropriate dimensions. Let $x(t ; \xi)$ denote the state trajectory of the neural network (5) from the initial data $x(\theta)=\xi(\theta)$ on $-h \leq \theta \leq 0$ in $L_{\mathcal{F}_{0}}^{2}\left([-h, 0] ; \mathbb{R}^{n}\right)$. It can be easily seen that the system (5) admits a trivial solution $x(t ; 0) \equiv 0$ corresponding to the initial data $\xi=0$, see [5], [11].

Remark 1: The parameter uncertainty structure as in (7)-(8) has been widely exploited in the problems of robust control and robust filtering of uncertain systems (see, e.g., [23], [25] and the references therein). Many practical systems possess parameter uncertainties which can be either exactly modeled or overbounded by (8). Observe that the unknown matrix $F$ in (7) can even be allowed to be state-dependent, i.e., $F(t)=F(t, x(t))$, as long as (8) is satisfied.

Remark 2: The assumption (9) on the stochastic disturbance term, $\sigma^{T}(t, x(t), x(t-h))$, has been used in recent papers dealing with stochastic neural networks, see [14] and references therein.

We are now in a position to introduce the notion of robust global asymptotic stability for the stochastic neural network (5) with parameter uncertainties and time-delays.

Definition 1: For the neural network (5) and every $\xi \in L_{\mathcal{F}_{0}}^{2}\left([-h, 0] ; \mathbb{R}^{n}\right)$, the trivial solution (equilibrium point) is robustly, globally, asymptotically stable in the mean square if, for all admissible uncertainties satisfying (8), the following holds:

$$
\lim _{t \rightarrow \infty} \mathbb{E}|x(t ; \xi)|^{2}=0
$$

The main purpose of the rest of this paper is to establish LMI-based stability criteria, which can be readily checked by using the Matlab LMI toolbox, such that the global asymptotic stability is guaranteed for the neural network (5) with both discrete and distributed time delays.

\section{MAIN RESULTS AND PROOFS}

We first give the following lemmas that are useful in deriving our LMI-based stability criteria.

Lemma 1: Let $x \in \mathbb{R}^{n}, y \in \mathbb{R}^{n}$ and $\varepsilon>0$. Then we have $x^{T} y+y^{T} x \leq \varepsilon x^{T} x+\varepsilon^{-1} y^{T} y$. Proof: The proof follows from the inequality $\left(\varepsilon^{1 / 2} x-\varepsilon^{-1 / 2} y\right)^{T}\left(\varepsilon^{1 / 2} x-\varepsilon^{-1 / 2} y\right) \geq 0$ immediately. 
Lemma 2: ( $S$-procedure) [25] Let $M=M^{T}, H$ and $E$ be real matrices of appropriate dimensions, with $F$ satisfying (8), then

$$
M+H F E+E^{T} F^{T} H^{T}<0,
$$

if and only if there exists a positive scalar $\varepsilon>0$ such that

$$
M+\frac{1}{\varepsilon} H H^{T}+\varepsilon E^{T} E<0,
$$

or equivalently

$$
\left[\begin{array}{ccc}
M & H & \varepsilon E^{T} \\
H^{T} & -\varepsilon I & 0 \\
\varepsilon E & 0 & -\varepsilon I
\end{array}\right]<0 .
$$

Lemma 3: [4] Given constant matrices $\Omega_{1}, \Omega_{2}, \Omega_{3}$ where $\Omega_{1}=\Omega_{1}^{T}$ and $0<\Omega_{2}=\Omega_{2}^{T}$, then

$$
\Omega_{1}+\Omega_{3}^{T} \Omega_{2}^{-1} \Omega_{3}<0
$$

if and only if

$$
\left[\begin{array}{cc}
\Omega_{1} & \Omega_{3}^{T} \\
\Omega_{3} & -\Omega_{2}
\end{array}\right]<0, \quad \text { or } \quad\left[\begin{array}{cc}
-\Omega_{2} & \Omega_{3} \\
\Omega_{3}^{T} & \Omega_{1}
\end{array}\right]<0 .
$$

Lemma 4: [10] For any positive definite matrix $M>0$, scalar $\gamma>0$, vector function $\omega:[0, \gamma] \rightarrow \mathbb{R}^{n}$ such that the integrations concerned are well defined, the following inequality holds:

For the sake of presentation simplicity, we denote:

$$
\left(\int_{0}^{\gamma} \omega(s) d s\right)^{T} M\left(\int_{0}^{\gamma} \omega(s) d s\right) \leq \gamma\left(\int_{0}^{\gamma} \omega^{T}(s) M \omega(s) d s\right)
$$

$$
\begin{aligned}
& \hat{M}:=\left[\begin{array}{ccccccc}
-A P-P A+\rho\left(\Sigma_{1}^{T} \Sigma_{1}+\Sigma_{2}^{T} \Sigma_{2}\right) & P W_{0} & \varepsilon_{1} G_{0}^{T} & P W_{1} & \varepsilon_{2} G_{1}^{T} & P W_{2} & \varepsilon_{3} \tau G_{2}^{T} \\
W_{0}^{T} P & -\varepsilon_{1} I & 0 & 0 & 0 & 0 & 0 \\
\varepsilon_{1} G_{0} & 0 & -\varepsilon_{1} I & 0 & 0 & 0 & 0 \\
W_{1}^{T} P & 0 & 0 & -\varepsilon_{2} I & 0 & 0 & 0 \\
\varepsilon_{2} G_{1} & 0 & 0 & 0 & -\varepsilon_{2} I & 0 & 0 \\
W_{2}^{T} P & 0 & 0 & 0 & 0 & -\varepsilon_{3} I & 0 \\
\varepsilon_{3} \tau G_{2} & 0 & 0 & 0 & 0 & 0 & -\varepsilon_{3} I
\end{array}\right], \\
& \hat{H}:=\left[\begin{array}{ccccccc}
-P M_{A} & P M_{0} & 0 & P M_{1} & 0 & P M_{2} & 0 \\
0 & 0 & 0 & 0 & 0 & 0 & 0 \\
\vdots & \vdots & \vdots & \vdots & \vdots & \vdots & \vdots \\
0 & 0 & 0 & 0 & 0 & 0 & 0
\end{array}\right] \text {, } \\
& \hat{F}:=\operatorname{diag}\{F, F, \cdots, F\} \text {, } \\
& \hat{E}:=\operatorname{diag}\left\{N_{A}, N_{0}, 0, N_{1}, 0, N_{2}, 0\right\},
\end{aligned}
$$

where $\hat{H}, \hat{F}$ and $\hat{E}$ have the same block structure as $\hat{M}$.

We are now ready to derive the conditions under which the network dynamics of (5) is robustly, globally, asymptotically stable in the mean square. The main theorem given below shows that the stability criteria can be expressed in terms of the feasibility of two linear matrix inequalities. 
Theorem 1: If there exist positive scalars $\rho>0, \varepsilon_{i}>0(i=1,2,3,4)$ and a positive definite matrix $P>0$ such that the following two linear matrix inequalities

$$
\begin{gathered}
P<\rho I, \\
{\left[\begin{array}{ccc}
\hat{M} & \hat{H} & \varepsilon_{4} \hat{E}^{T} \\
\hat{H}^{T} & -\varepsilon_{4} I & 0 \\
\varepsilon_{4} \hat{E} & 0 & -\varepsilon_{4} I
\end{array}\right]<0}
\end{gathered}
$$

hold, where $\hat{M}, \hat{H}, \hat{E}$ are defined in (11), (12), and (14), respectively, then the dynamics of the neural network (5) is robustly, globally, asymptotically stable in the mean square.

Proof: First of all, we know from the $S$-procedure (Lemma 2) that, there exists a positive scalar $\varepsilon_{4}>0$ such that (16) holds if and only if there exists a positive scalar $\varepsilon_{4}>0$ such that

$$
\hat{M}+\varepsilon_{4}^{-1} \hat{H} \hat{H}^{T}+\varepsilon_{4} \hat{E}^{T} \hat{E}<0 .
$$

Since $\hat{F}^{T} \hat{F} \leq I$ where $\hat{F}$ is defined in (13), it follows from

$$
\left(\varepsilon_{4}^{-1 / 2} \hat{H} \hat{F}-\varepsilon_{4}^{1 / 2} \hat{E}^{T}\right)\left(\varepsilon_{4}^{-1 / 2} \hat{H} \hat{F}-\varepsilon_{4}^{1 / 2} \hat{E}^{T}\right)^{T} \geq 0
$$

that

$$
\hat{H} \hat{F} \hat{E}+\hat{E}^{T} \hat{F}^{T} \hat{H}^{T} \leq \varepsilon_{4}^{-1} \hat{H} \hat{H}^{T}+\varepsilon_{4} \hat{E}^{T} \hat{E},
$$

and therefore (17) implies

$$
\hat{M}+\hat{H} \hat{F} \hat{E}+\hat{E}^{T} \hat{F}^{T} \hat{H}^{T}<0 .
$$

In terms of (11)-(14), after tedious algebraic manipulations, (18) can be rewritten as

$$
\left[\begin{array}{ccccccc}
-\bar{A} P-P \bar{A}+\rho\left(\Sigma_{1}^{T} \Sigma_{1}+\Sigma_{2}^{T} \Sigma_{2}\right) & P \bar{W}_{0} & \varepsilon_{1} G_{0}^{T} & P \bar{W}_{1} & \varepsilon_{2} G_{1}^{T} & P \bar{W}_{2} & \varepsilon_{3} \tau G_{2}^{T} \\
\bar{W}_{0}^{T} P & -\varepsilon_{1} I & 0 & 0 & 0 & 0 & 0 \\
\varepsilon_{1} G_{0} & 0 & -\varepsilon_{1} I & 0 & 0 & 0 & 0 \\
\bar{W}_{1}^{T} P & 0 & 0 & -\varepsilon_{2} I & 0 & 0 & 0 \\
\varepsilon_{2} G_{1} & 0 & 0 & 0 & -\varepsilon_{2} I & 0 & 0 \\
\bar{W}_{2}^{T} P & 0 & 0 & 0 & 0 & -\varepsilon_{3} I & 0 \\
\varepsilon_{3} \tau G_{2} & 0 & 0 & 0 & 0 & 0 & -\varepsilon_{3} I
\end{array}\right],
$$

and $\bar{A}, \bar{W}_{0}, \bar{W}_{1}$ and $\bar{W}_{2}$ are defined in (6)-(7).

Pre- and post-multiplying the inequality (19) by the block-diagonal matrix

$$
\operatorname{diag}\left\{I, \varepsilon_{1}^{-1 / 2} I, \varepsilon_{1}^{-1 / 2} I, \varepsilon_{2}^{-1 / 2} I, \varepsilon_{2}^{-1 / 2} I, \varepsilon_{3}^{-1 / 2} I, \varepsilon_{3}^{-1 / 2} I\right\}
$$

yield

$\left[\begin{array}{ccccccc}-\bar{A} P-P \bar{A}+\rho\left(\Sigma_{1}^{T} \Sigma_{1}+\Sigma_{2}^{T} \Sigma_{2}\right) & \varepsilon_{1}^{-1 / 2} P \bar{W}_{0} & \varepsilon_{1}^{1 / 2} G_{0}^{T} & \varepsilon_{2}^{-1 / 2} P \bar{W}_{1} & \varepsilon_{2}^{1 / 2} G_{1}^{T} & \varepsilon_{3}^{-1 / 2} P \bar{W}_{2} & \varepsilon_{3}^{1 / 2} \tau G_{2}^{T} \\ \varepsilon_{1}^{-1 / 2} \bar{W}_{0}^{T} P & -I & 0 & 0 & 0 & 0 & 0 \\ \varepsilon_{1}^{1 / 2} G_{0} & 0 & -I & 0 & 0 & 0 & 0 \\ \varepsilon_{2}^{-1 / 2} \bar{W}_{1}^{T} P & 0 & 0 & -I & 0 & 0 & 0 \\ \varepsilon_{2}^{1 / 2} G_{1} & 0 & 0 & 0 & -I & 0 & 0 \\ \varepsilon_{3}^{1 / 2} \bar{W}_{2} P & 0 & 0 & 0 & 0 & -I & 0 \\ \varepsilon_{3}^{-1 / 2} \tau G_{2} & 0 & 0 & 0 & 0 & 0 & -I\end{array}\right]<0$,


or

$$
\left[\begin{array}{cc}
\Omega_{1} & \Omega_{3}^{T} \\
\Omega_{3} & -\Omega_{2}
\end{array}\right]<0,
$$

where

$$
\begin{aligned}
& \Omega_{1}:=-\bar{A} P-P \bar{A}+\rho\left(\Sigma_{1}^{T} \Sigma_{1}+\Sigma_{2}^{T} \Sigma_{2}\right) \\
& \Omega_{2}:=I \\
& \Omega_{3}:=\left[\begin{array}{llllll}
\varepsilon_{1}^{-1 / 2} P \bar{W}_{0} & \varepsilon_{1}^{1 / 2} G_{0}^{T} & \varepsilon_{2}^{-1 / 2} P \bar{W}_{1} & \varepsilon_{2}^{1 / 2} G_{1}^{T} & \varepsilon_{3}^{-1 / 2} P \bar{W}_{2} & \varepsilon_{3}^{1 / 2} \tau G_{2}^{T}
\end{array}\right]^{T} .
\end{aligned}
$$

It follows from the Schur Complement Lemma (Lemma 3) that (21) holds if and only if

$$
\Omega_{1}+\Omega_{3}^{T} \Omega_{2}^{-1} \Omega_{3}<0
$$

or

$$
\begin{gathered}
-\bar{A} P-P \bar{A}+\rho\left(\Sigma_{1}^{T} \Sigma_{1}+\Sigma_{2}^{T} \Sigma_{2}\right)+\varepsilon_{1}^{-1} P \bar{W}_{0} \bar{W}_{0}^{T} P+\varepsilon_{1} G_{0}^{T} G_{0}+\varepsilon_{2}^{-1} P \bar{W}_{1} \bar{W}_{1}^{T} P \\
+\varepsilon_{2} G_{1}^{T} G_{1}+\varepsilon_{3}^{-1} P \bar{W}_{2} \bar{W}_{2}^{T} P+\varepsilon_{3} \tau^{2} G_{2}^{T} G_{2}<0 .
\end{gathered}
$$

In other words, from the condition of Theorem 1, we arrive at the conclusion that there exist positive scalars $\rho>0$, $\varepsilon_{i}>0(i=1,2,3)$ and a positive definite matrix $P>0$ such that (22) is true. Also, it follows immediately from (4) that

$$
l_{i}^{T}(x) l_{i}(x) \leq\left|G_{i} x\right|^{2}=x^{T} G_{i}^{T} G_{i} x
$$

Define a Lyapunov-Krasovskii functional candidate $V(t, x(t)) \in \mathcal{C}^{2,1}\left(\mathbb{R}^{+} \times \mathbb{R}^{n} ; \mathbb{R}^{+}\right)$by

$$
V(t, x(t))=x^{T}(t) P x(t)+\int_{t-h}^{t} x^{T}(s) Q_{1} x(s) d s+\int_{-\tau}^{0} \int_{t+s}^{t} x^{T}(\eta) Q_{2} x^{T}(\eta) d \eta d s,
$$

where $P$ is the positive definite solution to the inequality (22), and $Q_{1} \geq 0$ and $Q_{2} \geq 0$ are defined by

$$
Q_{1}:=\varepsilon_{2} G_{1}^{T} G_{1}+\rho \Sigma_{2}^{T} \Sigma_{2}, \quad Q_{2}:=\varepsilon_{3} \tau G_{2}^{T} G_{2} .
$$

By Itô's differential formula (see, e.g., [8]), the stochastic derivative of $V(t, x(t))$ along (5) can be obtained as follows:

$$
\begin{aligned}
d V(t, x(t))= & \left\{x^{T}(t)\left(-\bar{A} P-P \bar{A}+Q_{1}+\tau Q_{2}\right) x(t)+x^{T}(t) P \bar{W}_{0} l_{0}(x(t))+l_{0}^{T}(x(t)) \bar{W}_{0}^{T} P x(t)\right. \\
& +\operatorname{trace}\left[\sigma^{T}(t, x(t), x(t-h)) P \sigma(t, x(t), x(t-h))\right] \\
& +x^{T}(t) P \bar{W}_{1} l_{1}(x(t-h))+l_{1}^{T}(x(t-h)) \bar{W}_{1}^{T} P x(t) \\
& -x^{T}(t-h) Q_{1} x(t-h)+x^{T}(t) P \bar{W}_{2} \int_{t-\tau}^{t} l_{2}(x(s)) d s \\
& \left.+\left(\int_{t-\tau}^{t} l_{2}(x(s)) d s\right)^{T} \bar{W}_{2}^{T} P x(t)-\int_{t-\tau}^{t} x^{T}(s) Q_{2} x(s) d s\right\} d t \\
& +\left[x^{T}(t) P \sigma(t, x(t), x(t-h))+\sigma^{T}(t, x(t), x(t-h)) P x(t)\right] d w(t) .
\end{aligned}
$$


Next, it follows from the conditions (9) and (15) that

$$
\begin{aligned}
& \operatorname{trace}\left[\sigma^{T}(t, x(t), x(t-h)) P \sigma(t, x(t), x(t-h))\right] \\
& \leq \lambda_{\max }(P) \operatorname{trace}\left[\sigma^{T}(t, x(t), x(t-h)) \sigma(t, x(t), x(t-h))\right] \\
& \leq \rho\left[x^{T}(t) \Sigma_{1}^{T} \Sigma_{1} x(t)+x^{T}(t-h) \Sigma_{2}^{T} \Sigma_{2} x(t-h)\right]
\end{aligned}
$$

For the positive scalars $\varepsilon_{1}>0, \varepsilon_{2}>0, \varepsilon_{3}>0$, it follows from Lemma 1 and (4) that

$$
\begin{gathered}
x^{T}(t) P \bar{W}_{0} l_{0}(x(t))+l_{0}^{T}(x(t)) \bar{W}_{0}^{T} P x(t) \\
\leq \varepsilon_{1} l_{0}^{T}(x(t)) l_{0}(x(t))+\varepsilon_{1}^{-1} x^{T}(t) P \bar{W}_{0} \bar{W}_{0}^{T} P x(t) \\
\leq x^{T}(t)\left(\varepsilon_{1} G_{0}^{T} G_{0}+\varepsilon_{1}^{-1} P \bar{W}_{0} W_{0}^{T} P\right) x(t), \\
x^{T}(t) P \bar{W}_{1} l_{1}(x(t-h))+l_{1}^{T}(x(t-h)) \bar{W}_{1}^{T} P x(t) \\
\leq \varepsilon_{2} l_{1}^{T}(x(t-h)) l_{1}(x(t-h))+\varepsilon_{2}^{-1} x^{T}(t) P \bar{W}_{1} \bar{W}_{1}^{T} P x(t) \\
\leq \varepsilon_{2} x^{T}(t-h) G_{1}^{T} G_{1} x(t-h)+\varepsilon_{2}^{-1} x^{T}(t) P \bar{W}_{1} \bar{W}_{1}^{T} P x(t),
\end{gathered}
$$

and

$$
\begin{gathered}
x^{T}(t) P \bar{W}_{2} \int_{t-\tau}^{t} l_{2}(x(s)) d s+\left(\int_{t-\tau}^{t} l_{2}(x(s)) d s\right)^{T} \bar{W}_{2}^{T} P x(t) \\
\leq \varepsilon_{3}\left(\int_{t-\tau}^{t} l_{2}(x(s)) d s\right)^{T} \int_{t-\tau}^{t} l_{2}(x(s)) d s+\varepsilon_{3}^{-1} x^{T}(t) P \bar{W}_{2} \bar{W}_{2}^{T} P x(t) .
\end{gathered}
$$

Furthermore, it can be seen from (4), (25) and Lemma 4 that

$$
\begin{gathered}
\varepsilon_{3}\left(\int_{t-\tau}^{t} l_{2}(x(s)) d s\right)^{T} \int_{t-\tau}^{t} l_{2}(x(s)) d s \leq \varepsilon_{3} \tau \int_{t-\tau}^{t} l_{2}^{T}(x(s)) l_{2}(x(s)) d s \\
\leq \varepsilon_{3} \tau \int_{t-\tau}^{t} x^{T}(s) G_{2}^{T} G_{2} x(s) d s=\int_{t-\tau}^{t} x^{T}(s) Q_{2} x(s) d s
\end{gathered}
$$

Using (25) and (27)-(31), we obtain from (26) that

$$
\begin{aligned}
d V(t, x(t)) \leq & \left\{x ^ { T } ( t ) \left[-\bar{A} P-P \bar{A}+\rho\left(\Sigma_{1}^{T} \Sigma_{1}+\Sigma_{2}^{T} \Sigma_{2}\right)+\varepsilon_{1}^{-1} P \bar{W}_{0} \bar{W}_{0}^{T} P+\varepsilon_{1} G_{0}^{T} G_{0}\right.\right. \\
& \left.\left.+\varepsilon_{2}^{-1} P \bar{W}_{1} \bar{W}_{1}^{T} P+\varepsilon_{2} G_{1}^{T} G_{1}+\varepsilon_{3}^{-1} P \bar{W}_{2} \bar{W}_{2}^{T} P+\varepsilon_{3} \tau^{2} G_{2}^{T} G_{2}\right] x(t)\right\} d t \\
& +\left[x^{T}(t) P \sigma(t, x(t), x(t-h))+\sigma^{T}(t, x(t), x(t-h)) P x(t)\right] d w(t) \\
= & x^{T}(t) \Pi x(t) d t+\left[x^{T}(t) P \sigma(t, x(t), x(t-h))+\sigma^{T}(t, x(t), x(t-h)) P x(t)\right] d w(t) .
\end{aligned}
$$

where $\Pi$ is defined as

$$
\begin{aligned}
\Pi:= & -\bar{A} P-P \bar{A}+\rho\left(\Sigma_{1}^{T} \Sigma_{1}+\Sigma_{2}^{T} \Sigma_{2}\right)+\varepsilon_{1}^{-1} P \bar{W}_{0} \bar{W}_{0}^{T} P+\varepsilon_{1} G_{0}^{T} G_{0} \\
& +\varepsilon_{2}^{-1} P \bar{W}_{1} \bar{W}_{1}^{T} P+\varepsilon_{2} G_{1}^{T} G_{1}+\varepsilon_{3}^{-1} P \bar{W}_{2} \bar{W}_{2}^{T} P+\varepsilon_{3} \tau^{2} G_{2}^{T} G_{2} .
\end{aligned}
$$

From (22), we know that $\Pi<0$. Taking the mathematical expectation of both sides of (32), we have

$$
\frac{d \mathbb{E} V(t, x(t))}{d t} \leq \mathbb{E}\left(x^{T}(t) \Pi x(t)\right) \leq-\lambda_{\min }(-\Pi) \mathbb{E}|x(t)|^{2} .
$$


It can now be concluded from Lyapunov stability theory that the dynamics of the neural network (5) is robustly, globally, asymptotically stable in the mean square. This completes the proof of Theorem 1.

Remark 3: In Theorem 1, the matrix inequalities (15)(16) are linear on the parameters $\rho>0, \varepsilon_{i}>0(i=1,2,3,4)$ and $P>0$. Therefore, by using the Matlab LMI toolbox, it is straightforward to check the feasibility of (15)(16) without tuning any parameters. Different from the existing results based on matrix norm computation, such as those given in [1], the LMI approach developed in this paper is numerically more efficient [4]. The LMI Control Toolbox implements state-of-the-art interior-point LMI solvers. While these solvers are significantly faster than classical convex optimization algorithms, it should be kept in mind that the complexity of LMI computations remains higher than that of solving, say, a Riccati equation. For instance, problems with a thousand design variables typically take over an hour on today's workstations [4]. However, research on LMI optimization is a very active area in the applied math, optimization and the operations research community, and substantial speed-ups can be expected in the future.

Remark 4: Note that Lemma 1 is used in the proof of Theorem 1 to tackle the parameter uncertainties, hence certain conservatism might be introduced. Such conservatism can be significantly reduced by selecting proper scalar parameters $\varepsilon$, see [26] for more details. It is worth pointing out that, following the similar line of [22], it is not difficult to prove the exponential stability (in the mean square) of the neural network (5) under same conditions in Theorem 1.

In the sequel, in order to demonstrate the usefulness of our main results, we consider three special cases as well as a more general case. The proofs of all the subsequent corollaries follow easily from Theorem 1, and are therefore omitted.

Case 1: We first consider the uncertainty-free case. That is, there are no parameter uncertainties, and the neural network is described by

$$
\begin{aligned}
d x(t)= & {\left[-A x(t)+W_{0} l_{0}(x(t))+W_{1} l_{1}(x(t-h))+W_{2} \int_{t-\tau}^{t} l_{2}(x(s)) d s\right] d t } \\
& +\sigma(t, x(t), x(t-h)) d w(t) .
\end{aligned}
$$

In this case, we have the following corollary.

Corollary 1: If there exist positive scalars $\rho>0, \varepsilon_{i}>0(i=1,2,3)$ and a positive definite matrix $P>0$ such that the LMIs $P<\rho I$ and

$$
\left[\begin{array}{ccccccc}
-A P-P A+\rho\left(\Sigma_{1}^{T} \Sigma_{1}+\Sigma_{2}^{T} \Sigma_{2}\right) & P W_{0} & \varepsilon_{1} G_{0}^{T} & P W_{1} & \varepsilon_{2} G_{1}^{T} & P W_{2} & \varepsilon_{3} \tau G_{2}^{T} \\
W_{0}^{T} P & -\varepsilon_{1} I & 0 & 0 & 0 & 0 & 0 \\
\varepsilon_{1} G_{0} & 0 & -\varepsilon_{1} I & 0 & 0 & 0 & 0 \\
W_{1}^{T} P & 0 & 0 & -\varepsilon_{2} I & 0 & 0 & 0 \\
\varepsilon_{2} G_{1} & 0 & 0 & 0 & -\varepsilon_{2} I & 0 & 0 \\
W_{2}^{T} P & 0 & 0 & 0 & 0 & -\varepsilon_{3} I & 0 \\
\varepsilon_{3} \tau G_{2} & 0 & 0 & 0 & 0 & 0 & -\varepsilon_{3} I
\end{array}\right]<0
$$

hold, then the neural network (35) is globally asymptotically stable in the mean square.

Corollary 1 is still believed to be new since there are few results on the stability analysis for stochastic systems with both discrete and distributed delays.

Case 2. We now consider the case where there is neither parameter uncertainty nor the distributed delay, and the neural network can be further reduced to

$$
d x(t)=\left[-A x(t)+W_{0} l_{0}(x(t))+W_{1} l_{1}(x(t-h))\right] d t+\sigma(t, x(t), x(t-h)) d w(t) .
$$


This case has been studied by several authors, for example, in [3], [14], where different assumptions on the function $\sigma(t, x(t), x(t-h))$ have been made. The following corollary offers an alternative criterion for testing the mean square stability of the delayed neural network (37).

Corollary 2: If there exist positive scalars $\rho>0, \varepsilon_{i}>0(i=1,2)$ and a positive definite matrix $P>0$ such that the LMIs $P<\rho I$ and

$$
\left[\begin{array}{ccccc}
-A P-P A+\rho\left(\Sigma_{1}^{T} \Sigma_{1}+\Sigma_{2}^{T} \Sigma_{2}\right) & P W_{0} & \varepsilon_{1} G_{0}^{T} & P W_{1} & \varepsilon_{2} G_{1}^{T} \\
W_{0}^{T} P & -\varepsilon_{1} I & 0 & 0 & 0 \\
\varepsilon_{1} G_{0} & 0 & -\varepsilon_{1} I & 0 & 0 \\
W_{1}^{T} P & 0 & 0 & -\varepsilon_{2} I & 0 \\
\varepsilon_{2} G_{1} & 0 & 0 & 0 & -\varepsilon_{2} I
\end{array}\right]<0
$$

hold, then the neural network (37) is globally asymptotically stable in the mean square.

Case 3. Assume that there is no parameter uncertainty, and there is only the distributed delay. In this case, the neural network can be simplified as

$$
d x(t)=\left[-A x(t)+W_{0} l_{0}(x(t))+W_{2} \int_{t-\tau}^{t} l_{2}(x(s)) d s\right] d t+\sigma(t, x(t), x(t-h)) d w(t),
$$

If we do not consider the stochastic effects, i.e., $\sigma(t, x(t), x(t-h))=0$, the neural network (39) has been extensively studied, see e.g. [18], [29], [30]. Hence, the following corollary still stands as a significant result.

Corollary 3: If there exist positive scalars $\rho>0, \varepsilon_{i}>0(i=1,2)$ and a positive definite matrix $P>0$ such that the LMIs $P<\rho I$ and

$$
\left[\begin{array}{ccccc}
-A P-P A+\rho\left(\Sigma_{1}^{T} \Sigma_{1}+\Sigma_{2}^{T} \Sigma_{2}\right) & P W_{0} & \varepsilon_{1} G_{0}^{T} & P W_{2} & \varepsilon_{2} \tau G_{2}^{T} \\
W_{0}^{T} P & -\varepsilon_{1} I & 0 & 0 & 0 \\
\varepsilon_{1} G_{0} & 0 & -\varepsilon_{1} I & 0 & 0 \\
W_{2}^{T} P & 0 & 0 & -\varepsilon_{2} I & 0 \\
\varepsilon_{2} \tau G_{2} & 0 & 0 & 0 & -\varepsilon_{2} I
\end{array}\right]<0
$$

hold, then the neural network (39) is globally asymptotically stable in the mean square.

Case 4. Finally, we like to show that our results can also be extended to the multiple-delay case without major difficulties. Consider now the following uncertain neural network with multiple delays and stochastic perturbations:

$$
\begin{aligned}
d x(t)= & {\left[-\bar{A} x(t)+\bar{W}_{0} l_{0}(x(t))+\sum_{i=1}^{m} \bar{W}_{1} l_{1}\left(x\left(t-h_{i}\right)\right)+\sum_{j=1}^{m} \bar{W}_{2} \int_{t-\tau_{j}}^{t} l_{2}(x(s)) d s\right] d t } \\
& +\sigma\left(t, x(t), x\left(t-h_{1}\right), \cdots, x\left(t-h_{m}\right)\right) d w(t),
\end{aligned}
$$

where the meanings of the network parameters are similar to those in (5), except that the function $\sigma(t, x(t), x(t-$ $\left.\left.h_{1}\right), \cdots, x\left(t-h_{m}\right)\right)$ is required to satisfy

$$
\operatorname{trace}\left[\sigma^{T}\left(t, x(t), x\left(t-h_{1}\right), \cdots, x\left(t-h_{m}\right)\right) \sigma\left(t, x(t), x\left(t-h_{1}\right), \cdots, x\left(t-h_{m}\right)\right)\right] \leq \sum_{i=0}^{m}\left|\Sigma_{i} x\left(t-h_{i}\right)\right|^{2} .
$$

In order to study the mean square stability of the neural network (41), we can modify the Lyapunov-Krasovskii functional candidate in (24) as follows

$$
V(t, x(t))=x^{T}(t) P x(t)+\sum_{i=1}^{m} \int_{t-h_{i}}^{t} x^{T}(s) Q_{1 i} x(s) d s+\sum_{j=1}^{m} \int_{-\tau_{j}}^{0} \int_{t+s}^{t} x^{T}(\eta) Q_{2 j} x^{T}(\eta) d \eta d s,
$$


and then along the same line of the proof of Theorem 1, it is not difficult to obtain similar results.

\section{NUMERICAL EXAMPLES}

Two simple examples are presented here in order to illustrate the usefulness of our main results. Our aim is to examine the global asymptotic stability of a given delayed stochastic neural network.

Example 1. In this example, we consider a two-neuron stochastic neural network (35) with both discrete and distributed delays but without parameter uncertainties, where

$$
\begin{gathered}
A=\left[\begin{array}{cc}
0.9 & 0 \\
0 & 0.9
\end{array}\right], W_{0}=\left[\begin{array}{cc}
1.2 & -1.5 \\
-1.7 & 1.2
\end{array}\right], W_{1}=\left[\begin{array}{ll}
1.1 & 0.5 \\
0.5 & 0.8
\end{array}\right], W_{2}=\left[\begin{array}{ll}
0.6 & 0.1 \\
0.1 & 0.2
\end{array}\right], \\
G_{0}=\left[\begin{array}{cc}
0.1 & 0 \\
0 & 0.2
\end{array}\right], G_{1}=\left[\begin{array}{cc}
0.2 & 0 \\
0 & 0.2
\end{array}\right], G_{2}=\left[\begin{array}{cc}
0.1 & 0 \\
0 & 0.1
\end{array}\right], \\
\Sigma_{1}=\left[\begin{array}{cc}
0.08 & 0 \\
0 & 0.08
\end{array}\right], \Sigma_{2}=\left[\begin{array}{cc}
0.09 & 0 \\
0 & 0.09
\end{array}\right], \tau=0.12 .
\end{gathered}
$$

By using the Matlab LMI toolbox, we solve the LMIs $P<\rho I$ and (36) for $\rho>0, \varepsilon_{1}>0, \varepsilon_{2}>0, \varepsilon_{3}>0, P>0$ and obtain

$$
\rho=1.1814, \quad \varepsilon_{1}=1.2365, \quad \varepsilon_{2}=0.9586, \quad \varepsilon_{3}=0.8806, \quad P=\left[\begin{array}{ll}
0.2294 & 0.0896 \\
0.0896 & 0.2495
\end{array}\right] .
$$

Therefore, it follows from Corollary 1 that the two-neuron neural network (35) is globally asymptotically stable in the mean square.

Example 2. Now, let us consider a third-order delayed stochastic neural network (5) with parameter uncertainties. The network data are given as follows:

$$
\begin{gathered}
A=\left[\begin{array}{ccc}
1.1 & 0 & 0 \\
0 & 1.2 & 0 \\
0 & 0 & 1.3
\end{array}\right], W_{0}=\left[\begin{array}{ccc}
0.3 & -1.8 & 0.5 \\
-1.1 & 1.6 & 1.1 \\
0.6 & 0.4 & -0.3
\end{array}\right], \\
W_{1}=\left[\begin{array}{ccc}
0.8 & 0.2 & 0.1 \\
0.2 & 0.6 & 0.6 \\
-0.8 & 1.1 & -1.2
\end{array}\right], W_{2}=\left[\begin{array}{ccc}
0.5 & 0.2 & 0.1 \\
0.3 & 0.7 & -0.3 \\
1.2 & -1.1 & -0.5
\end{array}\right], \\
G_{0}=G_{1}=G_{2}=0.2 I_{3}, \Sigma_{1}=\Sigma_{2}=0.08 I_{3}, \tau=0.1, \\
M_{A}=N_{A}=M_{i}=N_{i}=0.1 I_{3}(i=0,1,1)
\end{gathered}
$$

Again, by solving the LMIs (15)-(16) for $\rho>0, \varepsilon_{i}>0(i=1,2,3,4)$ and $P>0$, we obtain

$$
\begin{gathered}
\rho=1.5648, \quad \varepsilon_{1}=1.2806, \quad \varepsilon_{2}=0.9575, \quad \varepsilon_{3}=0.9954, \quad \varepsilon_{4}=0.99868, \\
P=\left[\begin{array}{ccc}
0.3017 & 0.0644 & 0.0304 \\
0.0644 & 0.2465 & 0.0240 \\
0.0304 & 0.0240 & 0.2294
\end{array}\right],
\end{gathered}
$$

which indicates that the delayed stochastic neural network (5) is robustly, globally, asymptotically stable in the mean square. 


\section{CONCLusions}

In this paper, we have dealt with the problem of global asymptotic stability analysis for a class of uncertain stochastic delayed neural networks, which involve both discrete and distributed time delays. We have removed the traditional monotonicity and smoothness assumptions on the activation function. A linear matrix inequality (LMI) approach has been developed to solve the problem addressed. The stability criteria have been derived in terms of the positive definite solution to an LMI involving several scalar parameters, and simple examples have been used to demonstrate the usefulness of the main results. One of the future research topics would be extend the present results to more general cases, for example, the case that the delays are time-varying, including both discrete and distributed delays, the case when the stability criteria are delay-dependent, and the case that exponential stability is investigated. The results will appear in the near future.

\section{REFERENCES}

[1] S. Arik, Stability analysis of delayed neural networks. IEEE Transactions on Circuits Systems -I, vol. 47, pp. 1089-1092, 2000.

[2] S. Arik, Global robust stability analysis of neural networks with discrete time delays, Chaos, Solitons and Fractals, vol. 26, no. 5, pp. 1407$1414,2005$.

[3] S. Blythe, X. Mao and X. Liao, Stability of stochastic delay neural networks, Journal of the Franklin Institute, vol. 338, pp. 481-495, 2001.

[4] S. Boyd, L. EI Ghaoui, E. Feron and V. Balakrishnan, Linear Matrix Inequalities in System and Control Theory. Philadelphia, PA: SIAM, 1994.

[5] T. Burton, Stability and periodic solution of ordinary differential equation and functional differential equations. Orlando, FL: Academic, 1985.

[6] J. Cao, A. Chen and X. Huang, Almost periodic attractor of delayed neural networks with variable coefficients, Physics Letters A, vol. 340, pp. 104-120, 2005.

[7] J. Cao and J. Wang, Global exponential stability and periodicity of recurrent neural networks with time delays, IEEE Trans. Circuits and Systems: Part I, vol. 52, no. 5, pp. 920-931, 2005.

[8] A. Friedman, Stochastic differential equations and their applications, New York, Academic Press, 1976.

[9] P. Gahinet, A. Nemirovsky, A. J. Laub and M. Chilali, LMI Control Toolbox: For Use with Matlab. The Math Works, Inc. 1995.

[10] K. Gu, An integral inequality in the stability problem of time-delay systems. In: Proceedings of 39th IEEE Conference on Decision and Control, December 2000, Sydney, Australia, pp. 2805-2810, 2000.

[11] J. K. Hale, Theory of functional differential equations. New York: Springer-Verlag, 1977.

[12] J. J. Hopfield, Neural networks and physical systems with emergent collect computational abilities, Proc. Nat. Acad. Sci., USA, vol. 79, no. 2, pp. 2554-2558, 1982.

[13] J. J. Hopfield, Neurons with graded response have collective computational properties like those of two-state neurons, Proc. Nat. Acad. Sci., USA, vol. 81, pp. 3088-3092, 1984.

[14] H. Huang, D. W. C. Ho and J. Lam, Stochastic stability analysis of fuzzy Hopfield neural networks with time-varying delays, IEEE Trans. Circuits and Systems: Part II, vol. 52, no. 5, pp. 251-255, May 2005.

[15] M. P. Joy, Results concerning the absolute stability of delayed neural networks, Neural Networks, vol. 13, pp. 613-616, 2000.

[16] G. Joya, M. A. Atencia and F. Sandoval, Hopfield neural networks for optimization: study of the different dynamics, Neurocomputing, vol. 43, pp. 219-237, 2002.

[17] W. J. Li and T. Lee, Hopfield neural networks for affine invariant matching, IEEE Trans. Neural Networks, vol. 12, pp. 1400-1410, 2001.

[18] J. Liang and J. Cao, Global asymptotic stability of bi-directional associative memory networks with distributed delays, Applied Mathematics and Computation, vol. 152, pp. 415-424, 2004.

[19] J. C. Principle, J.-M. Kuo and S. Celebi, An analysis of the gamma memory in dynamic neural networks, IEEE Trans. Neural Networks, vol. 5, no. 2, pp. 337-361, 1994.

[20] S. Ruan and R. S. Filfil, Dynamics of a two-neuron system with discrete and distributed delays. Physica D, vol. 191, pp. 323-342, 2004.

[21] D. W. Tank and J. J. Hopfield, Neural computation by concentrating information in time, Proc. Nat. Acad. Sci., vol. 84, pp. 1896-1991, 1987. 
[22] Z. Wang and D. W. C. Ho, Filtering on nonlinear time-delay stochastic systems, Automatica, vol. 39, no. 1, pp. 101-109, 2003.

[23] Z. Wang, D. W. C. Ho and X. Liu, Variance-constrained filtering for uncertain stochastic systems with missing measurements, IEEE Trans. Automat. Control, vol. 48, pp. 1254-1258, July 2003.

[24] Z. Wang, Y. Liu and X. Liu, On global asymptotic stability of neural networks with discrete and distributed delays, Physics Letters A, vol. 345, no. 4-6, pp. 299-308, 2005.

[25] L. Xie, Output feedback $H_{\infty}$ control of systems with parameter uncertainty, Int. J. Control, vol. 63, pp. 741-750, 1996.

[26] L. Xie and Y. C. Soh, Robust Kalman filtering for uncertain systems, Systems \& Control Letters, vol. 22, pp. 123-129, 1994.

[27] S. Xu, J. Lam and D. W. C. Ho, Novel global robust stability criteria for interval neural networks with multiple time-varying delays, Physics Letters A, vol. 342, no. 4, pp. 322-330, 2005.

[28] S. Young, P. Scott and N. Nasrabadi, Object recognition using multilayer Hopfield neural network, IEEE Trans. Image Processing, vol. 6, no. 3, pp. 357-372, 1997

[29] H. Zhao, Global asymptotic stability of Hopfield neural network involving distributed delays. Neural Networks, vol. 17, pp. 47-53, 2004.

[30] H. Zhao,Existence and global attractivity of almost periodic solution for cellular neural network with distributed delays. Applied Mathematics and Computation, vol. 154, pp. 683-695, 2004. 